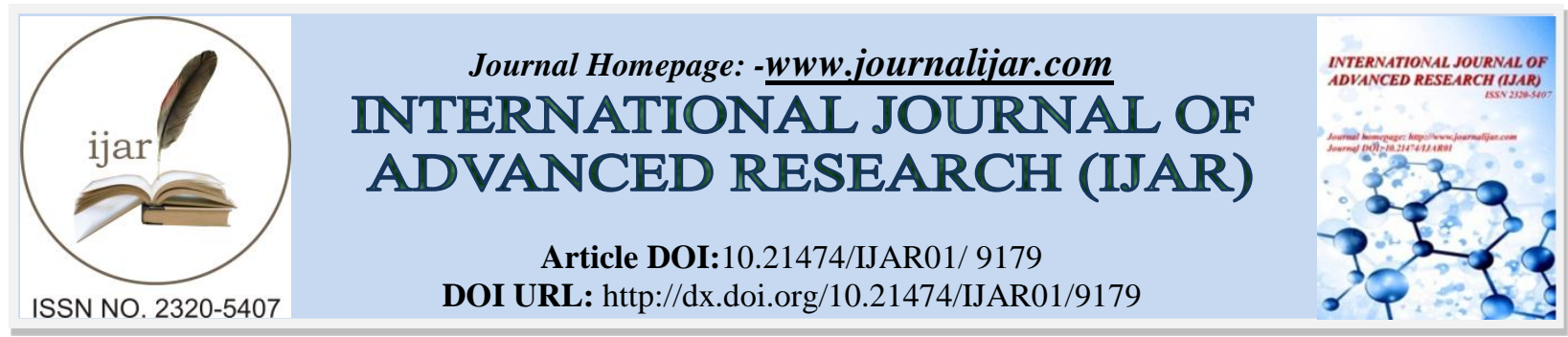

RESEARCH ARTICLE

\title{
THE EFFECT OF SOIL AMENDMENT TO NUTRIENT UPTAKE OF MAIZE AND YIELD IN THE SECOND PLANTING SEASON.
}

Kurnia Sari ${ }^{1}$, Bakhtiar Basyah ${ }^{2}$ And Zaitun².

1. Master Student of Magister Agroecotechnology, Faculty of Agriculture, Universitas Syiah Kuala, Darussalam, Banda Aceh, 23111, Indonesia.

2. Department of Agroecotechnology, Faculty of Agriculture, Universitas Syiah Kuala, Darussalam, Banda Aceh, 23111, Indonesia.

\section{Manuscript Info}

[..........................

Manuscript History

Received: 26 March 2019

Final Accepted: 28 April 2019

Published: May 2019

Key words:-

rice husk biochar, rice husk, maize yield, nutrient uptake.

\begin{abstract}
One of the potential soil texture for agriculture activity was sandy soil. But, there was needed some technology to improve the quality of sandy soil especially physical and chemical properties. Biochar was a stable carbon product which produced from the pyrolysis processed of organic matter. The addition of biochar was expected to be an alternative solution for physical, chemical properties and increase soil organic carbon and water availability. The experimental was aimed to determine the effect of soil amendment from rice husk, rice husk biochar, and cow manure to nutrient uptake of maize and yield of maize during the second planting season. The research was conducted in ACIAR experimental site, Aceh Besar district, Aceh province, Indonesia. The experimental design was completely randomized design non-factorial with three replications. The rates of soil amendment were rice husk biochar $5 \mathrm{tha}^{-1}$ and $10 \mathrm{tha}^{-1}$, rice husk $5 \mathrm{tha}^{-1}$ and $10 \mathrm{tha}^{-1}$, and cow manure $10 \mathrm{t} \mathrm{ha}^{-1}$ and $20 \mathrm{t} \mathrm{ha}^{-1}$. The rates of soil amendment before was given the addition fertilizer like $\mathrm{N}$ and $\mathrm{K}, 50 \% \mathrm{NPK}$ fertilizer recommendations and without NPK as a control. The results showed that the rates of $20 \mathrm{tha}^{-1}$ cow manure added $\mathrm{N}$ and $\mathrm{K}$ fertilizer was giving the best effect to maize growth, the rates of $10 \mathrm{t} \mathrm{ha}^{-1}$ cow manure added $\mathrm{N}$ and $\mathrm{K}$ fertilizer was giving the best effect to maize yield, and the rates of $10 \mathrm{t} \mathrm{ha}^{-1}$ and $5 \mathrm{tha}^{-1}$ soil amendment with the addition of $\mathrm{N}$ and $\mathrm{K}$ fertilizer, and the rates of $10 \mathrm{t} \mathrm{ha}^{-1}$ cow manure with the addition of $\mathrm{N}$ and $\mathrm{K}$ were giving the best effect to $\mathrm{N}$ uptake. But, the application of soil amendment was not significant to $\mathrm{P}$ and $\mathrm{K}$ plant uptake
\end{abstract}

Copy Right, IJAR, 2019,. All rights reserved.

\section{Introduction:-}

Soil fertility was the main factor to provide the sufficient amounts of nutrients in available and balanced form to ensure optimum plant growth and production. Nutrient requirements needed by plants for growth and production are determined by the ability of the soil to provide nutrients to plants and cannot always be fulfilled. The field was used intensively without crop rotation will caused the depletion of essential nutrients from the soil during harvest and soil

Corresponding Author:-Kurnia Sari.

Address:-Master Student of Magister Agroecotechnology, Faculty of Agriculture, Universitas Syiah

Kuala. Darussalam. Banda Aceh. 23111. Indonesia. 
fertility will decrease continuously. The unfertil soil could be a major factor to soil productivity, so the addition of nutrients in the soil through fertilization was very important in order to obtain profitable agricultural production. The fertilization recomendation for maize was still impersonal, so fertilization still irrational and unbalanced. Farmers were use fertilizer excessively and other farmers use less fertilizer than what is needed by plants, so maize production was not optimal because there was nothing balance of nutrients in the soil. In other cases, the availability of chemical fertilizers is increasingly difficult and the price is more expensive, because of the reduction in subsidies by the government, so that their use must be more efficient. Balanced fertilization is one of the key factors for improving and increasing the productivity of agricultural land. The application of fertilizers to soil will add one or more soil nutrients and will change the balance of other nutrients (Silalahi et al., 2006). Until now the nutrients that still have problems are nitrogen, phosphorus and potassium because they are needed in large quantities and they are called primary macro nutrients. They often lack in soil (Hasibuan, 2006). The soil in this study was alluvial eutric type according to the Indonesian National Classification System (2014) or typical aqualluvent (entisol) according to USA land taxonomy (2014 land survey staff) and had deep soil solum $(>90 \mathrm{~cm}$ ). based on soil physical analysis that the soil has a medium soil texture (sandy clay) with rather moderate bulk density (1.06-1.24 Mg m-3) and has a low to moderate water holding capacity (12.83-16.72\%). Soil permeability and porosity are good but low porosity values and have a variety of soil aggregate stability indices (unstable to somewhat stable). Making biochar using raw materials in the form of plant residues such as agricultural crop residues. Differences in raw materials produce differences in the characteristics of the biochar content produced so that the quality of biochar also depends on the type of material and the characteristics of the ingredients used (Shenbagavalli and Mahimairaja, 2012). Biochar is made from incomplete combustion, leaving nutrients that make the soil fertile and can be used as one of the tillers (Gani, 2009). Biochar is used as ameliorant and not as fertilizer. Biochar has a high Cation Exchange Capacity (CEC) that can bind soil cations and used by plants.

\section{Material and Method:- Materials}

This research was using a crop rotation system during one year of planting. Which began in kale vegetable crops in March - April 2018, maize which began in May - August 2018, soybeans which began in September - November 2018, mustard vegetables in January 2019. The research was conducted in the ACIAR Project Experimental Site, Aceh Besar district, Aceh province, Indonesia from May 2018 to August 2018. The treatment of first soil amendment was composed of soil amendment namely rice husk biochar (without NPK fertilizer, N and K fertilizer, and 50\% NPK recommendation), and rice husk (without NPK, N and K fertilizers and 50\% NPK recommendation). The treatment of soil amendment in the first planting as residual treatment on the second planting in the dry season and on the same plot. This research was a field research followed by research in the laboratory with the following steps: (a) field experiments by planting Bima 20 varieties of maize, NPK fertilizers and soil amendment treatment; (b) nutrient uptake of maize plants $45 \mathrm{DAP}$, and maize crop products converted in tons. The experiment was arranged in a completely randomized design with non factorial consists of 63 experimental units with 21 treatments and 3 replications.

\section{Results and Discussion:-}

Table 1:-Plant height, number of leaves, wet weight and dry weight at 15, 30, 45, and 60 DAP and at harvest.

\begin{tabular}{|l|c|c|c|}
\hline \multirow{2}{*}{ Parameter 45 DAP } & \multicolumn{3}{|c|}{ Soil Amendment } \\
\cline { 2 - 4 } & Biochar Rice husk & Rice husk & Cow manure \\
\hline Plant height $(\mathrm{cm})$ & $(195.33-203.47) \mathrm{NS}$ & $(142.00-195.33) \mathrm{NS}$ & $(165.80-196.13) \mathrm{NS}$ \\
\hline Number of leaves & $(15.20-15.33) \mathrm{NS}$ & $(14.80-15.93) \mathrm{NS}$ & $(14.07-15.67) \mathrm{NS}$ \\
\hline Fresh weight $(\mathrm{g})$ & $(57.40-68.17) \mathrm{NS}$ & $(53.00-67.17) \mathrm{NS}$ & $(62.00-72.37) \mathrm{NS}$ \\
\hline Dry weight $(\mathrm{g})$ & $(11.11-14.43) \mathrm{NS}$ & $(11.53-15.60) \mathrm{NS}$ & $(12.40-15.20) \mathrm{NS}$ \\
\hline
\end{tabular}

Table 1. showed that the plant height at age 45 DAP or vegetative period shows that the type and the dosage of soil amendment are not significant as well as the number of leaves and wet weight and oven dry weight. But from the results of the calculation of soil enhancers which are more dominant or more give a good response, namely the provision of cow manure. Cow manure contains urine and gas, such as methane and ammonia. Nutrient content in cow dung varies depending on the level of production, type, amount of feed consumption, and individual livestock (Gani, 2009). The composition of cow manure which has generally been studied has an element of Hemisellulosa $18.6 \%$, Cellulose 25.2\%, Lignin 20.2\%, Protein 14.9\%, Dust 13\% (Candrasekhar, 2005). This shows that the addition of organic fertilizer can increase the organic content in the soil so that it can provide nutrients in the soil 
needed by plants. According to Djoehana (1986), the use of organic fertilizer is intended to increase soil organic matter content and improve soil physical properties, especially the structure and porosity of the soil so that the amount of nutrients needed by plants is more available (Wahyudi Prasetyo, 2013). This shows that the addition of $\mathrm{N}$ and $\mathrm{K}$ fertilizers can improve soil physical properties, soil chemical properties and soil bology properties which will directly result in plant growth, especially the formation of leaves. Nitrogen nutrients are needed during the plant growth phase, but are most needed at the beginning to mid phase of primordial flower tillers.

Table 2:-Leaf length, leaf width, cob length, cob diameter and cob position

\begin{tabular}{|c|c|c|c|c|c|}
\hline Treatment & $\begin{array}{c}\text { Leaf } \\
\text { length } \\
(\mathrm{cm})\end{array}$ & $\begin{array}{c}\text { Leaf } \\
\text { width } \\
(\mathrm{cm})\end{array}$ & $\begin{array}{l}\text { Cob length } \\
\quad(\mathrm{cm})\end{array}$ & $\begin{array}{c}\text { Cob } \\
\text { diameter } \\
(\mathbf{m m})\end{array}$ & $\begin{array}{c}\text { Cob } \\
\text { position } \\
(\mathbf{c m})\end{array}$ \\
\hline $\begin{array}{l}\text { R1 } \\
\text { (NIL) }\end{array}$ & $\begin{array}{c}75.53 \\
\text { bcd }\end{array}$ & $\begin{array}{c}9.13 \\
a b\end{array}$ & $\begin{array}{c}14.07 \\
\mathrm{ab}\end{array}$ & $\begin{array}{c}33.70 \\
\text { abc }\end{array}$ & $\begin{array}{l}74.60 \\
\text { bcd }\end{array}$ \\
\hline $\begin{array}{l}\mathrm{R} 2 \\
(\mathrm{~N} \text { and } \mathrm{K})\end{array}$ & $\begin{array}{c}80.40 \\
\text { bcd }\end{array}$ & $\begin{array}{l}9.93 \\
\text { bcde }\end{array}$ & $\begin{array}{l}18.37 \\
\text { fg }\end{array}$ & $\begin{array}{l}39.90 \\
\text { ef }\end{array}$ & $\begin{array}{l}79.33 \\
\text { bcde }\end{array}$ \\
\hline $\begin{array}{l}\text { R3 } \\
\text { (NPK } 50 \% \text { recomendation) }\end{array}$ & $\begin{array}{c}75.87 \\
\text { bcd }\end{array}$ & $\begin{array}{l}10.00 \\
\text { bcde }\end{array}$ & $\begin{array}{c}16.20 \\
\text { Abcdefg }\end{array}$ & $\begin{array}{l}37.33 \\
\text { abcdef }\end{array}$ & $\begin{array}{l}75.53 \\
\text { bcd }\end{array}$ \\
\hline 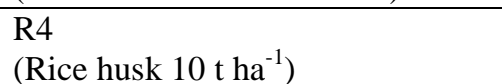 & $\begin{array}{c}59.40 \\
\mathrm{a}\end{array}$ & $\begin{array}{c}9.47 \\
\text { abcde }\end{array}$ & $\begin{array}{c}13.30 \\
\mathrm{a}\end{array}$ & $\begin{array}{c}32.40 \\
\mathrm{a}\end{array}$ & $\begin{array}{c}56.47 \\
\mathrm{a}\end{array}$ \\
\hline $\begin{array}{l}\text { R5 } \\
\left.\text { (Rice husk } 10 \mathrm{tha}^{-1}+\mathrm{NK}\right)\end{array}$ & $\begin{array}{c}72.23 \\
b\end{array}$ & $\begin{array}{l}9.27 \\
a b c\end{array}$ & $\begin{array}{c}18.23 \\
\text { efg }\end{array}$ & $\begin{array}{c}39.13 \\
\text { def }\end{array}$ & $\begin{array}{l}74.40 \\
\text { bcd }\end{array}$ \\
\hline $\begin{array}{l}\text { R6 } \\
\text { (Rice husk } 10 \mathrm{t} \mathrm{ha}^{-1}+\text { NPK } 50 \% \text { ) }\end{array}$ & $\begin{array}{c}74.33 \\
\text { bcd }\end{array}$ & $\begin{array}{l}9.40 \\
\text { abcd }\end{array}$ & $\begin{array}{c}15.67 \\
\text { abcdef }\end{array}$ & $\begin{array}{c}33.73 \\
\text { abc }\end{array}$ & $\begin{array}{c}73.67 \\
b\end{array}$ \\
\hline $\begin{array}{l}\text { R7 } \\
\text { (Rice husk } 5 \mathrm{tha}^{-1} \text { ) }\end{array}$ & $\begin{array}{c}74.93 \\
\text { bcd }\end{array}$ & $\begin{array}{l}9.27 \\
\mathrm{abc}\end{array}$ & $\begin{array}{c}14.07 \\
\mathrm{ab}\end{array}$ & $\begin{array}{l}34.13 \\
\text { abcd }\end{array}$ & $\begin{array}{l}75.33 \\
\text { bcd }\end{array}$ \\
\hline $\begin{array}{l}\text { R8 } \\
\text { (Rice husk } 5 \text { t/ha }+ \text { NK) }\end{array}$ & $\begin{array}{c}78.00 \\
\text { bcd }\end{array}$ & $\begin{array}{l}9.53 \\
\text { bcde }\end{array}$ & $\begin{array}{c}18.33 \\
\text { fg }\end{array}$ & $\begin{array}{c}39.53 \\
\text { ef }\end{array}$ & $\begin{array}{l}74.40 \\
\text { bcd }\end{array}$ \\
\hline $\begin{array}{l}\text { R9 } \\
\text { (Rice husk } 5 \text { t/ha + NPK } 50 \% \text { ) }\end{array}$ & $\begin{array}{c}78.87 \\
\text { bcd }\end{array}$ & $\begin{array}{l}9.40 \\
\text { abcd }\end{array}$ & $\begin{array}{c}16.73 \\
\text { bcdefg }\end{array}$ & $\begin{array}{l}38.50 \\
\text { cdef }\end{array}$ & $\begin{array}{l}76.53 \\
\text { bcde }\end{array}$ \\
\hline $\begin{array}{l}\text { R10 } \\
\text { (Biochar } 10 \text { t/ha) }\end{array}$ & $\begin{array}{c}73.13 \\
\text { bc }\end{array}$ & $\begin{array}{l}9.53 \\
\text { bcde }\end{array}$ & $\begin{array}{l}15.20 \\
\text { abcde }\end{array}$ & $\begin{array}{c}36.50 \\
\text { abcdef }\end{array}$ & $\begin{array}{l}79.73 \\
\text { bcde }\end{array}$ \\
\hline $\begin{array}{l}\text { R11 } \\
\text { (Biochar } 10 \mathrm{t} / \mathrm{ha}+\mathrm{NK})\end{array}$ & $\begin{array}{c}78.07 \\
\text { bcd }\end{array}$ & $\begin{array}{c}8.47 \\
\mathrm{a}\end{array}$ & $\begin{array}{c}18.17 \\
\text { efg }\end{array}$ & $\begin{array}{c}39.27 \\
\text { ef }\end{array}$ & $\begin{array}{l}78.13 \\
\text { bcde }\end{array}$ \\
\hline $\begin{array}{l}\text { R12 } \\
\text { (Biochar } 10 \mathrm{t} / \mathrm{ha}+\text { NPK } 50 \%)\end{array}$ & $\begin{array}{c}77.93 \\
\text { bcd }\end{array}$ & $\begin{array}{l}10.00 \\
\text { bcde }\end{array}$ & 17.07 bcdefg & $\begin{array}{r}34.80 \\
\text { abcde }\end{array}$ & $\begin{array}{l}78.07 \\
\text { bcde }\end{array}$ \\
\hline $\begin{array}{l}\text { R13 } \\
\text { (Biochar 5 t/ha) }\end{array}$ & $\begin{array}{c}75.13 \\
\text { bcd }\end{array}$ & $\begin{array}{l}9.53 \\
\text { bcde }\end{array}$ & $14.30 \mathrm{abc}$ & $\begin{array}{c}33.80 \\
\text { abc }\end{array}$ & $\begin{array}{l}80.87 \\
\text { bcde }\end{array}$ \\
\hline $\begin{array}{l}\text { R14 } \\
(\text { Biochar } 5 \text { t/ha }+ \text { NK) }\end{array}$ & $\begin{array}{c}76.37 \\
\text { bcd }\end{array}$ & $\begin{array}{l}9.87 \\
\text { bcde }\end{array}$ & 17.17 cdefg & $\begin{array}{l}38.17 \\
\text { bcdef }\end{array}$ & $\begin{array}{l}77.53 \\
\text { bcde }\end{array}$ \\
\hline $\begin{array}{l}\text { R14 } \\
\text { (Biochar } 5 \text { t/ha + NK) }\end{array}$ & $\begin{array}{c}76.37 \\
\text { bcd }\end{array}$ & $\begin{array}{l}9.87 \\
\text { bcde }\end{array}$ & 17.17 cdefg & $\begin{array}{l}38.17 \\
\text { bcdef }\end{array}$ & $\begin{array}{l}77.53 \\
\text { bcde }\end{array}$ \\
\hline $\begin{array}{l}\text { R15 } \\
\text { (Biochar } 5 \text { t/ha + NPK 50\%) }\end{array}$ & $\begin{array}{c}79.80 \\
\text { bcd }\end{array}$ & $\begin{array}{l}9.87 \\
\text { bcde }\end{array}$ & 16.80 bcdefg & $\begin{array}{l}37.90 \\
\text { bcdef }\end{array}$ & $\begin{array}{l}87.93 \\
\text { cde }\end{array}$ \\
\hline $\begin{array}{l}\text { R16 } \\
\text { (Cow manure } 10 \mathrm{t} / \mathrm{ha})\end{array}$ & $\begin{array}{c}76.73 \\
\text { bcd }\end{array}$ & $\begin{array}{c}10.47 \\
\text { def }\end{array}$ & $14.73 \mathrm{abcd}$ & $\begin{array}{c}33.27 \\
\mathrm{ab}\end{array}$ & $\begin{array}{l}78.80 \\
\text { bcde }\end{array}$ \\
\hline $\begin{array}{l}\text { R17 } \\
(\text { Cow manure } 10 \mathrm{t} / \mathrm{ha}+\mathrm{NK})\end{array}$ & $\begin{array}{c}82.53 \\
\mathrm{~d}\end{array}$ & $\begin{array}{c}10.60 \\
f\end{array}$ & $18.43 \mathrm{fg}$ & $\begin{array}{c}39.83 \\
\text { ef }\end{array}$ & $\begin{array}{c}88.73 \\
\text { de }\end{array}$ \\
\hline $\begin{array}{l}\text { R18 } \\
\text { (Cow manure } 10 \text { t/ha + NPK 50\%) }\end{array}$ & $\begin{array}{l}82.40 \\
\mathrm{~cd}\end{array}$ & $\begin{array}{l}10.27 \\
\text { cdef }\end{array}$ & 16.67 bcdefg & 36.13 abcdef & $\begin{array}{l}87.87 \\
\text { cde }\end{array}$ \\
\hline $\begin{array}{l}\text { R19 } \\
\text { (Cow manure } 20 \mathrm{t} / \mathrm{ha})\end{array}$ & $\begin{array}{c}81.67 \\
\mathrm{~cd}\end{array}$ & $\begin{array}{c}10.47 \\
\text { def }\end{array}$ & 15.80 abcdefg & 35.37 abcdef & $\begin{array}{l}84.67 \\
\text { bcde }\end{array}$ \\
\hline $\begin{array}{l}\text { R20 } \\
(\text { Cow manure } 20 \mathrm{t} / \mathrm{ha}+\mathrm{NK})\end{array}$ & $\begin{array}{c}83.20 \\
\mathrm{~d}\end{array}$ & $\begin{array}{c}10.53 \\
\text { ef }\end{array}$ & $\begin{array}{c}19.00 \\
\mathrm{~g}\end{array}$ & $\begin{array}{l}40.07 \\
\mathrm{f}\end{array}$ & $\begin{array}{c}89.60 \\
\mathrm{e}\end{array}$ \\
\hline $\begin{array}{l}\text { R21 } \\
\text { (Cow manure } 20 \text { t/ha + NPK 50\%) }\end{array}$ & $\begin{array}{l}82.27 \\
\mathrm{~cd}\end{array}$ & $\begin{array}{c}10.60 \\
f\end{array}$ & $\begin{array}{l}17.67 \\
\text { defg }\end{array}$ & $\begin{array}{c}38.50 \\
\text { cdef }\end{array}$ & $\begin{array}{l}88.07 \\
\text { cde }\end{array}$ \\
\hline
\end{tabular}


Table 2. Shows that the treatment of soil enhancers gives the best response to soil amendment of cow manure $20 \mathrm{t}$ ha -1 with the addition of $\mathrm{N}$ and $\mathrm{K}$ fertilizers only for parameters of leaf length, leaf width, cob diameter, cob length and cob position. However, the same does not apply significantly to the treatment of other land enhancers. The results of the variance analysis showed that the treatment of the combination of $\mathrm{N}$ and $\mathrm{K}$ fertilizers had a significant effect on leaf width in observation of R20 (20 t / ha + NK cow manure. Results of leaf width studies showed that the best value was found in treatment R20 (20 t / cow ha + NK Cow manure is an organic material that can be used as fertilizer in intensification.In addition to the content of $\mathrm{N}, \mathrm{P}$ and $\mathrm{K}$ contained in it is quite high, this organic material has not been utilized optimally by farmers. and improve the physical and chemical properties of the soil, but the same does not apply significantly to the treatment of other soil enhancers.

Tabel 3:-weight with maize husk

\begin{tabular}{|c|c|c|}
\hline Treatment & Weight with maize husk & Weight without maize husk \\
\hline $\begin{array}{l}\mathrm{R} 1 \\
(\mathrm{NIL})\end{array}$ & $154.61 \mathrm{a}$ & $136.43 \mathrm{ab}$ \\
\hline $\begin{array}{l}\mathrm{R} 2 \\
(\mathrm{~N} \text { and } \mathrm{K})\end{array}$ & $223.31 \mathrm{ab}$ & $199.58 \mathrm{abcd}$ \\
\hline $\begin{array}{l}\text { R3 } \\
\text { (NPK } 50 \% \text { recomendation) }\end{array}$ & $173.57 \mathrm{ab}$ & $162.26 \mathrm{abcd}$ \\
\hline $\begin{array}{l}\text { R4 } \\
\left(\text { Rice husk } 10 \mathrm{t} \mathrm{ha}^{-1}\right)\end{array}$ & $113.42 \mathrm{a}$ & $100.73 \mathrm{a}$ \\
\hline $\begin{array}{l}\text { R5 } \\
\left(\text { Rice husk } 10 \mathrm{t} \mathrm{ha}^{-1}+\mathrm{NK}\right)\end{array}$ & $228.68 \mathrm{ab}$ & $204.50 \mathrm{bcd}$ \\
\hline $\begin{array}{l}\text { R6 } \\
\left(\text { Rice husk } 10 \mathrm{t} \mathrm{ha}^{-1}+\text { NPK } 50 \%\right)\end{array}$ & $152.44 \mathrm{a}$ & $142.94 \mathrm{abc}$ \\
\hline 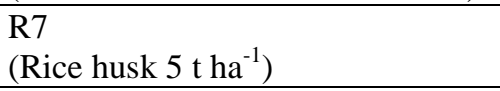 & $130.64 \mathrm{a}$ & $119.98 \mathrm{ab}$ \\
\hline $\begin{array}{l}\text { R8 } \\
\text { (Rice husk } 5 \text { t/ha }+ \text { NK) }\end{array}$ & $192.66 \mathrm{ab}$ & $207.03 \mathrm{bcd}$ \\
\hline $\begin{array}{l}\text { R9 } \\
(\text { Rice husk } 5 \text { t/ha + NPK } 50 \%)\end{array}$ & $184.3 \mathrm{ab}$ & $175.51 \mathrm{abcd}$ \\
\hline $\begin{array}{l}\text { R10 } \\
\text { (Biochar } 10 \text { t/ha) }\end{array}$ & $160.89 \mathrm{a}$ & $141.47 \mathrm{abc}$ \\
\hline $\begin{array}{l}\text { R11 } \\
(\text { Biochar } 10 \text { t/ha }+ \text { NK) }\end{array}$ & $235.81 \mathrm{ab}$ & $199.29 \mathrm{abcd}$ \\
\hline $\begin{array}{l}\text { R12 } \\
(\text { Biochar } 10 \text { t/ha + NPK } 50 \%)\end{array}$ & $178.51 \mathrm{ab}$ & $247.38 \mathrm{~d}$ \\
\hline $\begin{array}{l}\text { R13 } \\
\text { (Biochar } 5 \text { t/ha) }\end{array}$ & $386.59 \mathrm{~b}$ & $126.64 \mathrm{ab}$ \\
\hline $\begin{array}{l}\text { R14 } \\
(\text { Biochar } 5 \text { t/ha }+ \text { NK })\end{array}$ & $185.51 \mathrm{ab}$ & $174.64 \mathrm{abcd}$ \\
\hline $\begin{array}{l}\text { R14 } \\
(\text { Biochar } 5 \text { t/ha }+ \text { NK })\end{array}$ & $173.72 \mathrm{ab}$ & $154.03 \mathrm{abcd}$ \\
\hline $\begin{array}{l}\text { R15 } \\
(\text { Biochar } 5 \text { t/ha + NPK 50\%) }\end{array}$ & $140.09 \mathrm{a}$ & $111.65 \mathrm{ab}$ \\
\hline $\begin{array}{l}\text { R16 } \\
\text { (Cow manure } 10 \text { t/ha) }\end{array}$ & $228.87 \mathrm{ab}$ & $212.94 \mathrm{bcd}$ \\
\hline $\begin{array}{l}\text { R17 } \\
(\text { Cow manure } 10 \text { t/ha }+ \text { NK })\end{array}$ & $160.12 \mathrm{a}$ & $147.80 \mathrm{abcd}$ \\
\hline $\begin{array}{l}\text { R18 } \\
(\text { Cow manure } 10 \text { t/ha + NPK 50\%) }\end{array}$ & $151.55 \mathrm{a}$ & $142.47 \mathrm{abc}$ \\
\hline $\begin{array}{l}\text { R19 } \\
\text { (Cow manure } 20 \text { t/ha) }\end{array}$ & $259.12 \mathrm{ab}$ & $240.88 \mathrm{~cd}$ \\
\hline $\begin{array}{l}\text { R20 } \\
(\text { Cow manure } 20 \text { t/ha }+ \text { NK) }\end{array}$ & $212.16 \mathrm{ab}$ & $197.15 \mathrm{abcd}$ \\
\hline
\end{tabular}


Table 3. shows that the components of the non-significant effect occur in the weight of the globus cob, the fresh weight of the cob without clobot (Table 3). The results of the variance analysis showed that soil enhancing residues from biochar at a dose of $5 \mathrm{t}$ ha -1 gave the best response to the weights with kolobot. Whereas for weighting parameters without kolobot the treatment of soil enhancers of biochar $10 \mathrm{t}$ ha- 1 with the addition of NPK fertilizer $50 \%$ of recommendations gave the best response, but this was not significantly different from other treatments.

Table 4:-Average nutrient uptake of maize plants in 45 DAP from rice husk and biochar residue treatment (10 tons ha-1) absorption of nutrient uptake significantly to nitrogen nutrient elements. Average data is shown in the table.

\begin{tabular}{|c|c|c|c|}
\hline Treatment & Nitrogen & Phosfor & Kalium \\
\hline $\begin{array}{l}\text { R1 } \\
\text { (NIL) }\end{array}$ & $1.35 \mathrm{ab}$ & $0.12 \mathrm{a}$ & $1.13 \mathrm{a}$ \\
\hline $\begin{array}{l}\mathrm{R} 2 \\
(\mathrm{~N} \text { and } \mathrm{K})\end{array}$ & $1.96 \mathrm{~cd}$ & $0.14 \mathrm{a}$ & $1.11 \mathrm{a}$ \\
\hline $\begin{array}{l}\text { R3 } \\
\text { (NPK } 50 \% \text { recomendation) }\end{array}$ & $1.73 \mathrm{bcd}$ & $0.12 \mathrm{a}$ & $1.05 \mathrm{a}$ \\
\hline $\begin{array}{l}\text { R4 } \\
\left(\text { Rice husk } 10 \mathrm{tha}^{-1}\right)\end{array}$ & $1.17 \mathrm{a}$ & $0.13 \mathrm{a}$ & $1.01 \mathrm{a}$ \\
\hline $\begin{array}{l}\text { R5 } \\
\left.\text { (Rice husk } 10 \mathrm{t} \mathrm{ha}^{-1}+\mathrm{NK}\right)\end{array}$ & $2.05 \mathrm{~d}$ & $0.14 \mathrm{a}$ & $1.25 \mathrm{a}$ \\
\hline $\begin{array}{l}\text { R6 } \\
\text { (Rice husk } 10 \mathrm{t} \mathrm{ha}^{-1}+\text { NPK } 50 \% \text { ) }\end{array}$ & $1.91 \mathrm{~cd}$ & $0.16 \mathrm{a}$ & $1.10 \mathrm{a}$ \\
\hline $\begin{array}{l}\text { R7 } \\
\left(\text { Rice husk } 5 \mathrm{t} \mathrm{ha}^{-1}\right)\end{array}$ & $1.45 \mathrm{abc}$ & $0.14 \mathrm{a}$ & $1.08 \mathrm{a}$ \\
\hline $\begin{array}{l}\text { R8 } \\
\text { (Rice husk } 5 \text { t/ha }+ \text { NK) }\end{array}$ & $2.05 \mathrm{~d}$ & $0.14 \mathrm{a}$ & $1.12 \mathrm{a}$ \\
\hline $\begin{array}{l}\text { R9 } \\
\text { (Rice husk } 5 \text { t/ha + NPK } 50 \% \text { ) }\end{array}$ & $1.91 \mathrm{~cd}$ & $0.13 \mathrm{a}$ & $1.07 \mathrm{a}$ \\
\hline $\begin{array}{l}\text { R10 } \\
\text { (Biochar } 10 \text { t/ha) }\end{array}$ & $1.26 \mathrm{ab}$ & $0.11 \mathrm{a}$ & $1.06 \mathrm{a}$ \\
\hline $\begin{array}{l}\text { R11 } \\
(\text { Biochar } 10 \mathrm{t} / \mathrm{ha}+\mathrm{NK})\end{array}$ & $2.05 \mathrm{~d}$ & $0.12 \mathrm{a}$ & $1.16 \mathrm{a}$ \\
\hline $\begin{array}{l}\text { R12 } \\
\text { (Biochar } 10 \text { t/ha + NPK } 50 \%)\end{array}$ & $1.63 \mathrm{abcd}$ & $0.12 \mathrm{a}$ & $1.11 \mathrm{a}$ \\
\hline $\begin{array}{l}\text { R13 } \\
\text { (Biochar } 5 \mathrm{t} / \mathrm{ha})\end{array}$ & $1.35 \mathrm{ab}$ & $0.11 \mathrm{a}$ & $1.02 \mathrm{a}$ \\
\hline $\begin{array}{l}\text { R14 } \\
(\text { Biochar } 5 \text { t/ha + NK) }\end{array}$ & $1.68 \mathrm{abcd}$ & $0.12 \mathrm{a}$ & $1.04 \mathrm{a}$ \\
\hline $\begin{array}{l}\text { R14 } \\
(\text { Biochar } 5 \text { t/ha + NK) }\end{array}$ & $1.45 \mathrm{abc}$ & $0.11 \mathrm{a}$ & $1.00 \mathrm{a}$ \\
\hline $\begin{array}{l}\text { R15 } \\
\text { (Biochar 5 t/ha + NPK 50\%) }\end{array}$ & $1.59 \mathrm{abcd}$ & $0.10 \mathrm{a}$ & $0.93 \mathrm{a}$ \\
\hline $\begin{array}{l}\text { R16 } \\
\text { (Cow manure } 10 \text { t/ha) }\end{array}$ & $2.05 \mathrm{~d}$ & $0.12 \mathrm{a}$ & $1.03 \mathrm{a}$ \\
\hline $\begin{array}{l}\text { R17 } \\
\text { (Cow manure } 10 \mathrm{t} / \mathrm{ha}+\mathrm{NK})\end{array}$ & $1.63 \mathrm{abcd}$ & $0.12 \mathrm{a}$ & $1.01 \mathrm{a}$ \\
\hline $\begin{array}{l}\text { R18 } \\
(\text { Cow manure } 10 \text { t/ha }+ \text { NPK 50\%) }\end{array}$ & $1.73 \mathrm{bcd}$ & $0.11 \mathrm{a}$ & $0.98 \mathrm{a}$ \\
\hline $\begin{array}{l}\text { R19 } \\
\text { (Cow manure } 20 \text { t/ha) }\end{array}$ & $1.96 \mathrm{~cd}$ & $0.12 \mathrm{a}$ & $1.03 \mathrm{a}$ \\
\hline $\begin{array}{l}\text { R20 } \\
(\text { Cow manure } 20 \mathrm{t} / \mathrm{ha}+\mathrm{NK})\end{array}$ & $1.88 \mathrm{~cd}$ & $0.12 \mathrm{a}$ & $1.06 \mathrm{a}$ \\
\hline
\end{tabular}

Table 4. shows that soil enhancers can improve soil fertility despite improvements in soil chemical properties such as increasing $\mathrm{N}, \mathrm{P}$ and $\mathrm{K}$ and availability. With the increase in the availability of $\mathrm{N}, \mathrm{P}$, and $\mathrm{K}$, the plants will have enough nutrients and then the plant height will increase. Increased growth causes an improvement in soil chemical 
properties as an increase in $\mathrm{N}$ and $\mathrm{P}$ in the soil (Purnomo, 2009). The amount of nutrient uptake at 45 DAP showed that the absorption of nitrogen elements had a significantly different response and the best treatment was found in soil manure enhancers with a dose of $10 \mathrm{t}$ ha- 1 with the addition of $\mathrm{N}$ and $\mathrm{K}$ fertilizers which was $2.05 \%$ per plot and Nitrogen nutrient uptake the lowest was in the treatment of soil enhancers of $10 \mathrm{t}$ ha-1 rice husk without the addition of NPK fertilizer which was $1.17 \%$ per plot. While the phosphorus and potassium nutrients of soil amendment did not give a response that was significantly different because $\mathrm{P}$ was available on the research land which was already high enough and the potassium contained in the soil amendment itself required a long time in decomposition. Based on the study of martyrdom (2016) it was suggested that four levels of biochar $(0,5,10$, and $15 \mathrm{~g} \mathrm{~kg} \mathrm{-} \mathrm{1)} \mathrm{and} \mathrm{two} \mathrm{levels} \mathrm{of} \mathrm{inorganic} \mathrm{fertilizers} \mathrm{(} 0$ and local recommendations for maize) were randomly applied to a complete randomized design with four replications. Maize is grown for 45 days in a greenhouse using sandy Podzol. An increase in soil $\mathrm{pH}$ along with a decrease in $\mathrm{Al}$ can be exchanged. Fertilized soil significantly increases total $\mathrm{N}$, with a simultaneous decrease in soil $\mathrm{pH}$ due to nitrification $\mathrm{N}$. Positive changes do occur in the soil due to the application of biochar, which leads to a significant increase in yield of dry matter and high maize.

Table 5:-Results of maize plants $\left(\mathrm{t} \mathrm{ha}^{-1}\right)$

\begin{tabular}{|l|c|}
\hline \multicolumn{1}{|c|}{ Treatment } & Yield of Maize t ha $^{-1}$ \\
\hline Control (0\% NPK or no NPK ) & $7.26 \mathrm{abcde}$ \\
\hline Control (N and K) & $7.53 \mathrm{bcdef}$ \\
\hline Control (NPK NPK 50\% recommendation) & $7.30 \mathrm{abcde}$ \\
\hline Rice husk 10 t/ha + NPK 0\% or no NPK & $9.08 \mathrm{ab}$ \\
\hline Rice husk 10 t/ha + N and K & $7.06 \mathrm{cdef}$ \\
\hline Rice husk 10 t/ha + NPK 50\% recommendation & $5.56 \mathrm{a}$ \\
\hline Rice husk 5 t/ha + NPK 0\% or no NPK & $9.36 \mathrm{def}$ \\
\hline Rice husk 5 t/ha + N and K & $7.84 \mathrm{abcdef}$ \\
\hline Rice husk 5 t/ha + NPK 50\% recommendation & $6.70 \mathrm{abc}$ \\
\hline Biochar rice husk 10 t/ha + NPK 0\% or no NPK & $9.50 \mathrm{ef}$ \\
\hline Biochar rice husk 10 t/ha + N and K & $7.53 \mathrm{abcdef}$ \\
\hline Biochar Rice husk 10 t/ha + NPK 50\% rekomendasi & $6.14 \mathrm{ab}$ \\
\hline Biochar Rice husk 5 t/ha + NPK 0\% atau tanpa NPK & $7.93 \mathrm{abcdef}$ \\
\hline Biochar Rice husk 5 t/ha + N dan K & $7.68 \mathrm{abcdef}$ \\
\hline Biochar Rice husk 5 t/ha + NPK 50\% rekomendasi & $6.32 \mathrm{ab}$ \\
\hline Cow manure 10 t/ha + NPK 0\% atau tanpa NPK & $10.01 \mathrm{f}$ \\
\hline Cow manure 10 t/ha + N dan K & $6.75 \mathrm{abcd}$ \\
\hline Cow manure 10 t/ha + NPK 50\% rekomendasi & $6.31 \mathrm{ab}$ \\
\hline Cow manure 20 t/ha + NPK 0\% atau tanpa NPK & $9.12 \mathrm{cdef}$ \\
\hline Cow manure 20 t/ha + N dan K & $8.57 \mathrm{bcdef}$ \\
\hline Cow manure 20 t/ha + NPK 50\% rekomendasi & \\
\hline
\end{tabular}

Table 5. Shows that the yield of grain potential per hectare at the second planting at $14 \%$ moisture content indicates that the best treatment for cow manure improvement is $10 \mathrm{t}$ ha -1 with the addition of fertilizer $\mathrm{N}$ and $\mathrm{K}$ only and soil amendment treatment of biochar rice husk $10 \mathrm{t}$ ha -1 with the addition of $\mathrm{N}$ and $\mathrm{K}$ fertilizers only. Chan et al. (2007) say that the various experiments that have been conducted show that the biochar application has real agronomic benefits, but the results are not universal because other experiments show different results or have negative effects. This is because biochar has several properties depending on the raw material and various interactions between the type of biochar and the soil.

\section{Conclusion:-}

The results showed that the administration of soil enhancers in dry land had a significant effect on nitrogen nutrient uptake and the effect of soil enhancers treated with $\mathrm{N}$ and $\mathrm{K}$ fertilizers. However, there was no significant effect on maize production. The best growth of maize was found in the treatment of $5 \mathrm{t}$ ha- 1 rice husk with the addition of $\mathrm{N}$ and $\mathrm{K}$ fertilizers. The treatment of soil change did not significantly influence plant height 35, 45 and 90 days after planting (DAP). The combination of $\mathrm{N}$ and $\mathrm{K}$ fertilizer treatment with 5 tons ha- 1 rice husk is the best maize yield. Soil amendments on dry land have a significant effect on nitrogen uptake in both single factors and the interaction between uptake absorption of soil amendments with $\mathrm{N}$ and $\mathrm{K}$ Total uptake of nutrient uptake of 45 DAP. However, 
there is no significant effect on maize production. The best growth results and yields of maize were found in the treatment of $5 \mathrm{t}$ ha- 1 rice husk with fertilizer $\mathrm{N}$ and $\mathrm{K}$.

\section{Acknowledgements}

Thanks to The ACIAR Project (N0. SMCN/2012/103) Improving Soil and Water Management and Crop Productivity of Dryland Agriculture System of Aceh and New South Wales. Thanks to my supervisor Dr. Bakhtiar Basyah, SP, M.Si. and Dr. Zaitun, SP, M.Si.

\section{References:-}

1. Chan, K.Y., Van Z.L., Meszaros I., Downie A., and Joseph S. 2007. Agronomic values of greenwaste biochar as a soil amendment. Australian Journal of Soil Research 45 (8): 629-634.

2. Chandrasekhar, P N Pramada, L Praveen. 2005. Effect of Organic Acid Treatment on The Properties of Rice Huck Silica. J Mater Sci. 40 (2005): 6535-6544.

3. Djoehana. 1986. Pupuk dan pemupukan. UGM Press. Yogyakarta.

4. Gani, A. 2009. Biochar Utilizatiom for Agricultural Land Improvement. Iptek Tanaman Pangan (ISSN 19074263) Vol. 4 No. 1, Juli 2009. p:33-48.

5. Hasibuan, B.E. 2006. Fertilizer and Fertilization. USU-Press, Medan. Kaderi, H. 2004. Technique of Pellet Fertilizer Processing from Weed as Compound Fertilizer and Its Effect on Paddy Plant. Buletin Teknik Pertanian. Vol. 9. No. 2. 2004.

6. Indonesian National Classification System. 2014. Soil map information in Indonesia, Indonesia Center for Agricultural Land Resource Research and Development Indonesia. Bogor. Jawa Barat.

7. Kanton, R. A. L,_Buah, S. S. J. 2017. Soil Amendments and Rotation Effects on Soybean and Maize Growths and Soil Chemical Changes in Northern Ghana, International Journal of Agronomy,Volume 2017,

8. Manickam,T. Maizeelissen, G. Bachmann, R. Ibrahim, I. Mulder, J. and Hale,S. 2017. Biochar Application in Malaysian Sandy and Acid Sulfate Soils: Soil Amelioration Effects and Improved Crop Production over Two Cropping Seasons, Sustainability, 10.3390/su71215842, 7, 12,(16756-16770), (2015).

9. Pirngadi, K dan Abdulrachman. 2005. Effect of Compund Fertilizer NPK (15-15-15) on Growth and Yield of Irrigation Padi. J. Agrivigor 4 (3):188-197 Agustus 2005; ISSN: 1412-2286. Indonesian Centre for Rice Research, Sukamandi, Subang, West Java.

10. Purnomo, J. 2009. Effect of NPK Fertilizer on Paddy Yied Ciherang Variety and Inceptisol Soil Chemistry Propertie. Bogor.

11. Silalahi, F., Y. Saragih, A. Marpaung, R. Hutabarat, Karsina dan S. R. Purba. 2006. Final Report of NPK Fertilization Test on Fruit Plant. Fruit Plant Research Institution. Kebun Percobaan Tanaman Buah (KPTB), Berastagi. Medan.

12. Shenbagavalli, S. and Mahimairaja, S. 2012. Production and characterization of biochar from different biological wastes. International Journal of Plant, Animal, and Environmental Sciences 2 (1) : $197-201$.

13. Syuhada, AB and Shamsuddin, J. 2016. Biochar as soil amendment: Impact on chemical properties and maize nutrient uptake in a Podzol. Department of Land Management, Faculty of Agriculture, Universiti Putra Malaysia, Serdang, Selangor 43400, Malaysia.

14. Wahyudi, P. Mudji S., Tatik W. 2013. The effect of various dosages of organic and anorganic fertilizers on plant growth and yield of sweet corn. Jurusan Budidaya Pertanian. Fakultas Pertanian. Universitas Brawijaya. Malang. Indonesia.

15. Yusup P, Herru D. 2013. Effect of Raw Material and Dose Combination Against Biochar Soil Physical Properties sandy At Maize (Zea mays L.), Fakultas Pertanian, Universitas Jember (UNEJ), Jember. 